\title{
Generation of Parametric Equivalent-Area Targets for Design of Low-Boom Supersonic Concepts*
}

\author{
$\mathrm{Wu} \mathrm{Li}{ }^{1}$ \\ NASA Langley Research Center, Hampton, Virginia 23681, USA \\ Elwood Shields ${ }^{2}$ \\ Alliant Techsystems, Inc., Hampton, Virginia 23681, USA
}

\begin{abstract}
A tool with an Excel visual interface is developed to generate equivalent-area $\left(A_{e}\right)$ targets that satisfy the volume constraints for a low-boom supersonic configuration. The new parametric $A_{\mathrm{e}}$ target explorer allows users to interactively study the tradeoffs between the aircraft volume constraints and the low-boom characteristics (e.g., loudness) of the ground signature. Moreover, numerical optimization can be used to generate the optimal $\boldsymbol{A}_{\mathrm{e}}$ target for given $A_{\mathrm{e}}$ volume constraints. A case study is used to demonstrate how a generated lowboom $A_{\mathrm{e}}$ target can be matched by a supersonic configuration that includes a fuselage, wing, nacelle, pylon, aft pod, horizontal tail, and vertical tail. The low-boom configuration is verified by sonic-boom analysis with an off-body pressure distribution at three body lengths below the configuration.
\end{abstract}

\footnotetext{
${ }^{*}$ AIAA Paper for 49th AIAA Aerospace Sciences Meeting, January 2011, Orlando, Florida.

${ }^{1}$ Senior Research Engineer, Aeronautics Systems Analysis Branch, Mail Stop 442.

${ }^{2}$ Senior System Engineer, Aeronautics Systems Analysis Branch, Space Division.
} 


\section{Nomenclature}

$A_{\mathrm{e}} \quad=\quad$ equivalent area

$d p / p=$ (calculated pressure - freestream pressure)/(freestream pressure)

$x, y=$ coordinates of a point on the plane

\section{Introduction}

ESIGN of low-boom supersonic configurations is still in its infancy. Developing a configuration with a shaped

$\mathrm{D}$ ground signature that is propagated from computational fluid dynamics (CFD) off-body pressure distributions is extremely difficult. A promising path for developing a low-boom configuration is a multifidelity approach that (a) starts from a low-fidelity low-boom design, (b) refines the low-fidelity design with CFD equivalent-area $\left(A_{\mathrm{e}}\right)$ analysis, and (c) improves the design with sonic-boom analysis using CFD off-body pressure distributions. Steps (a) and (b), along with the analysis portion of step (c), have been implemented in ModelCenter [1] as a mixed-fidelity design process for low-boom supersonic concepts [2]. A design case study using this mixed-fidelity process is documented in reference [2].

A major gap in the previously mentioned mixed-fidelity design process is the lack of a tool to generate an $A_{\mathrm{e}}$ target that is appropriate for the low-boom concept. To fill this gap, we have developed an Excel-based $A_{\mathrm{e}} \operatorname{target}$ explorer. The new parametric $A_{\mathrm{e}}$ target explorer allows users to interactively study the tradeoffs between the aircraft volume constraints and the low-boom characteristics (e.g., loudness) of the ground signature. Moreover, numerical optimization can be used to generate the optimal $A_{\mathrm{e}}$ target for given $A_{\mathrm{e}}$ volume constraints. This approach is different from the existing target generation processes that use parametric F-functions [3]-[7]. The approach aims at eliminating the practical difficulties of using the design parameters of an F-function to obtain an $A_{\mathrm{e}}$ target that satisfies the volume constraints of a low-boom concept.

A case study is used to demonstrate how to match a low-boom $A_{\mathrm{e}}$ target with a supersonic configuration; the mixed-fidelity design process that is documented in reference [2] is used for the case study. The configuration is designed for a cruise weight of 30,000 lb, a cruise Mach number of 1.6, and a cruise altitude of 45,000 ft. Sonicboom analysis with CFD off-body pressure distributions is used to verify that the mixed-fidelity design has a shaped ground signature with perceived loudness of 82.3 PLdB. The off-track boom analysis for this configuration is given in reference [8].

This paper is organized as follows. Section II introduces the parametric $A_{\mathrm{e}}$ target explorer. The results for the tradeoff analysis between the $A_{\mathrm{e}}$ volume and the perceived loudness are included in Section III. The case study is provided in Section IV and concluding remarks are given in Section V.

\section{Parametric Equivalent-Area Target Explorer}

Seebass and Argrow [7] give a comprehensive review of the historical development of boom minimization theory and call it the classical Jones-Seebass-George-Darden theory. Existing theories for generating low-boom targets are based on parametric forms of the F-function [3]-[6], which can be converted to a low-boom target $A_{\mathrm{e}}$ (see ref. [9] for the relationships between the F-function and $A_{\mathrm{e}}$ ).

However, attaining certain characteristics for the $A_{\mathrm{e}}$ shape of a configuration with parametric F-functions is typically difficult (see ref. [6]). In contrast, a parametric $A_{\mathrm{e}}$ distribution provides direct control of the shape of the $\operatorname{target} A_{\mathrm{e}}$. As a result, we have developed an Excel-based parametric $A_{\mathrm{e}}$ target explorer (see Figure 1).

The $A_{\mathrm{e}}$ target explorer has seven command buttons that allow the following functions: read the input data, add/remove spline control points, update the $A_{\mathrm{e}}$ curve, update the analysis results, recover the previous target, and export the target $A_{\mathrm{e}}$.

The parametric $A_{\mathrm{e}}$ distribution is defined by the B-spline control points. The first control point is at $(0,0)$, and the last control point is determined by the equivalent length of the aircraft, the cruise speed, the cruise weight, and the cruise altitude. Each of the remaining control points can be selected as active by choosing the appropriate control point from a pull-down list. The $x$ and $y$ coordinates of the active control point can be moved by using the two scroll bars. The resulting spline curve is a B-spline interpolation of the control points with zero as the first derivative at the first and the last control point. 


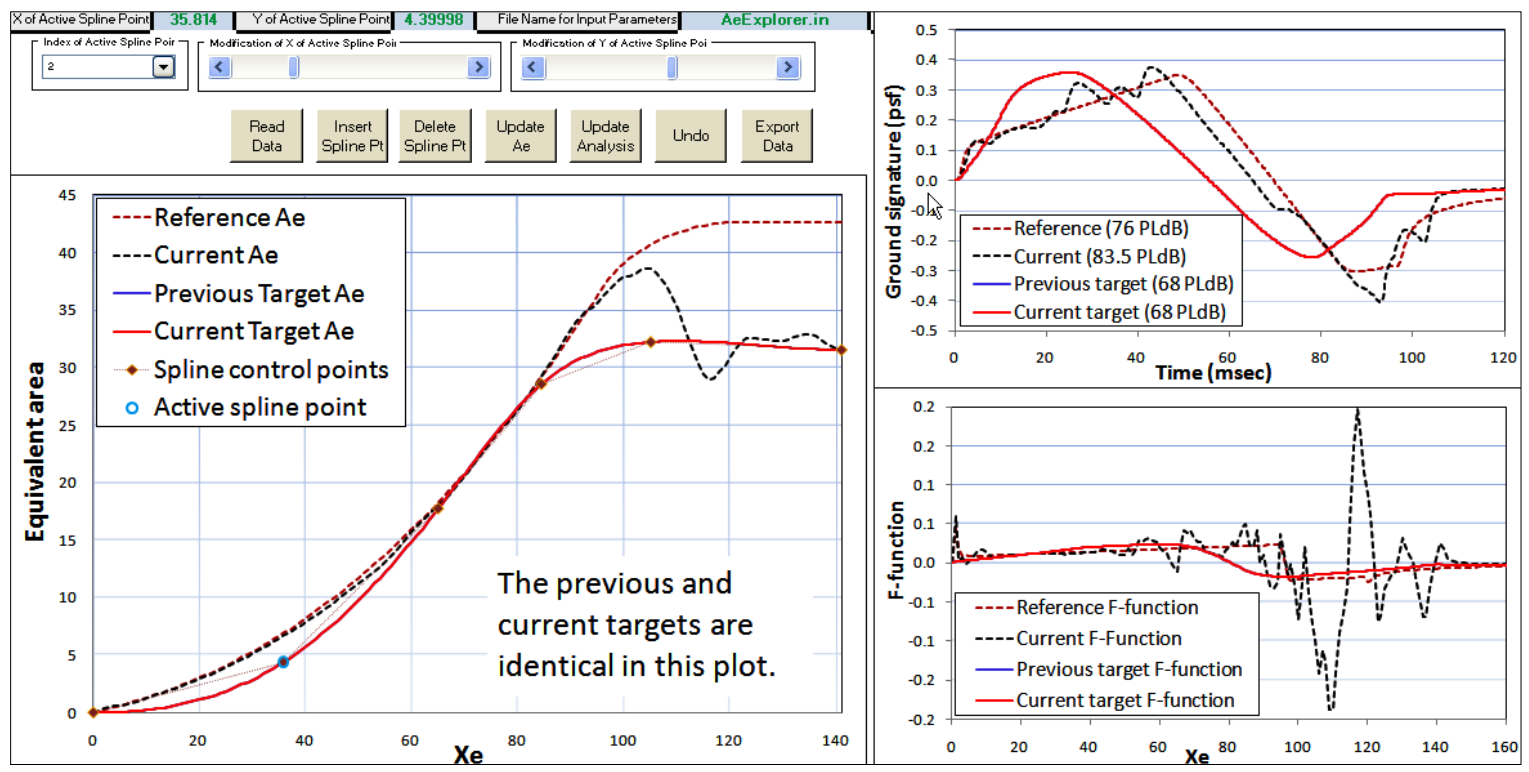

Figure 1. Excel visual interface for $A_{\mathrm{e}}$ target explorer.

The input data includes a reference $A_{\mathrm{e}}$, the $A_{\mathrm{e}}$ distribution for the current configuration, and the flight conditions. The reference $A_{\mathrm{e}}$ in Figure 1 is a classical target that was used to guide the layout and shaping of the initial configuration. The current $A_{\mathrm{e}}$ in Figure 1 is the $A_{\mathrm{e}}$ of the current configuration. A user can import an existing target $A_{\mathrm{e}}$ to be scaled to match the $A_{\mathrm{e}}$ of the current configuration at the end point as the initial target for the target exploration. After modifying the B-spline control points and updating the analysis results, the current target $A_{\mathrm{e}}$ becomes the previous target $A_{\mathrm{e}}$, and the current target $A_{\mathrm{e}}$ is updated by the new control points. The corresponding Ffunction and ground signature plots, along with the loudness data, are also included in the Excel interface. All the ground signatures in this paper are generated by the sonic-boom propagation code sBOOM [10].

The current $A_{\mathrm{e}}$ in Figure 1 shows that the $A_{\mathrm{e}}$ volume for the cockpit requires a minimum $A_{\mathrm{e}}$ value of approximately $16 \mathrm{ft}^{2}$ at $x=60 \mathrm{ft}$. To generate a classical reference target $A_{\mathrm{e}}$ with this constraint, the $A_{\mathrm{e}}$ value at the end must be larger than the value that is determined by the flight conditions. This volume requirement can be satisfied by keeping the $y$ coordinate of the $\mathrm{B}$-spline control point above 16 at around $x=60$; this is relatively easy to control during the target exploration process.

\section{Tradeoffs of Equivalent Area Targets}

A configuration that was derived from a public configuration of a Gulfstream low-boom supersonic demonstrator was used to generate the current $A_{\mathrm{e}}$ for the $A_{\mathrm{e}}$ target explorer as shown in Figure 1 . The $A_{\mathrm{e}}$ range for the configuration is $140 \mathrm{ft}$, with a cruise weight of $30,000 \mathrm{lb}$, a cruise Mach number of 1.6, and a cruise altitude of 45,000 ft. The ending $A_{\mathrm{e}}$ value (i.e., the $A_{\mathrm{e}}$ value at $x=140$ ) is $33.8 \mathrm{ft}^{2}$, which is determined by the flight conditions.

Six B-spline control points were shown to be adequate for the parametric $A_{\mathrm{e}}$ to obtain a ground signature with a sinusoidal shape. More control points could lead to an undesirable shape of the ground signature and make the interactive target exploration difficult to control. A sinusoidal signature was used by Howe for the development of a quiet spike low-boom concept (see fig. 15b in ref. [11]).

After moving the control points to the desired locations while maintaining an $A_{\mathrm{e}}$ value above 16 at $x=60$, we obtain the Smooth Target 3 that is shown in Figure 2. In the process, the PLdB value of the target signature is manually minimized by moving each control point in four directions (without violating the volume requirement at $x$ $=60$ ), respectively, until no reduction in the PLdB of the target signature can be achieved. Then, the volume constraint is enforced by placing bounds on the control parameters in the genetic optimizer in ModelCenter to minimize the PLdB of the ground signature; this yields an almost identical $A_{\mathrm{e}}$ target with a reduction of 0.1 in the PLdB value of the ground signature. 


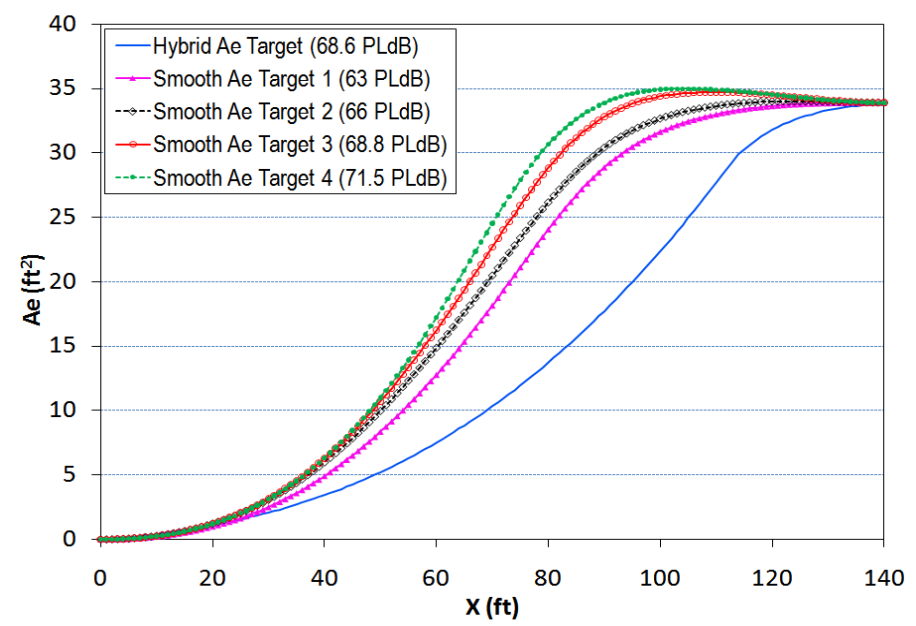

Figure 2. Tradeoffs between $A_{\mathrm{e}}$ volume and PLdB of corresponding ground signature.

To understand the tradeoffs between the $A_{\mathrm{e}}$ volume and the low-boom characteristics of the ground signature, we start to adjust the $A_{\mathrm{e}}$ volume of the Smooth Target 3. This leads to Smooth Targets 2 and 4, which are shown in Figure 2. Next, we remove the $A_{\mathrm{e}}$ volume requirement and determine the target $A_{\mathrm{e}}$ that has the ground signature with the lowest PLdB value. This is done by using a genetic optimization code, which generates Smooth Target 1. Finally, we use the hybrid low-boom target code [5], which is an extension of the classical low-boom target generation process, to generate a target for the specified flight conditions and aircraft length. The result is plotted in Figure 2 as the hybrid $A_{\mathrm{e}}$ target. The corresponding F-functions and ground signatures for these $A_{\mathrm{e}}$ targets are given in Figure 3.
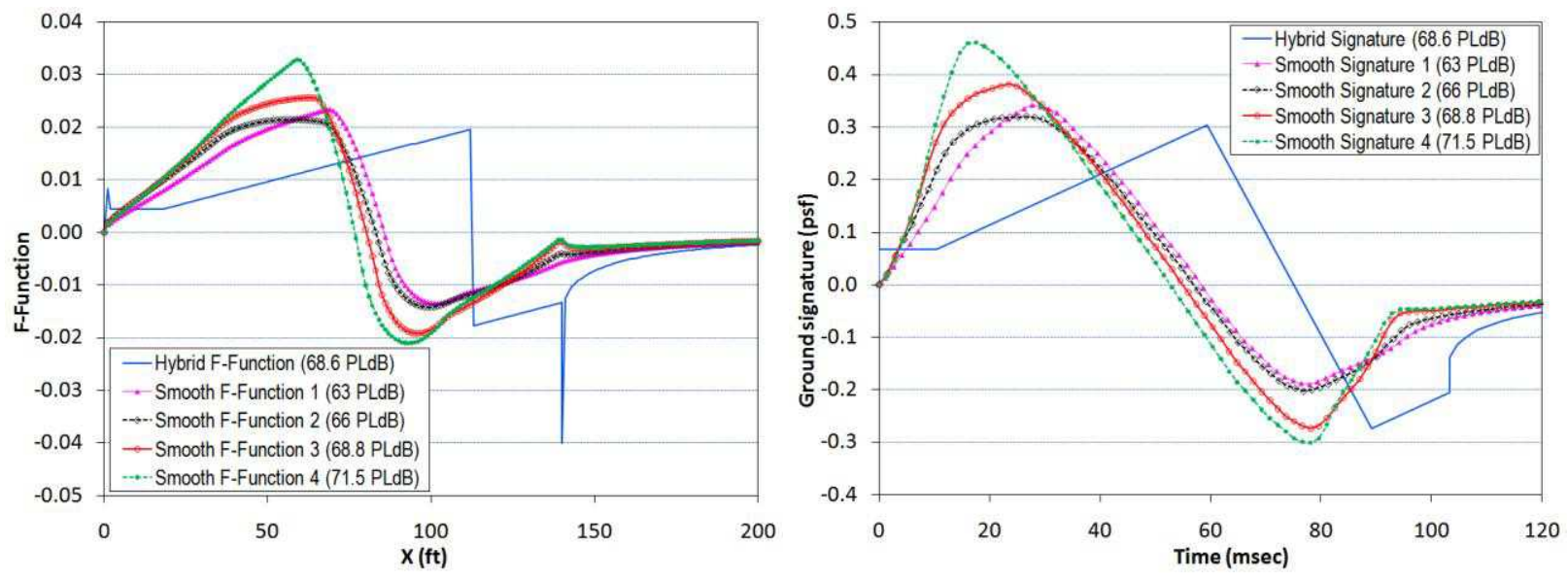

Figure 3. Corresponding F-functions and signatures for $A_{\mathrm{e}}$ tradeoffs.

The PLdB values for the smooth target signatures are 63, 66, 68.8, and 71.5, which indicate a trend of sonicboom penalty for $A_{\mathrm{e}}$ volume requirements. Also note that the $A_{\mathrm{e}}$ values for the hybrid $A_{\mathrm{e}}$ target are much lower than those for the smooth $A_{\mathrm{e}}$ target 1 , while the PLdB value is significantly higher. This result demonstrates the advantage to using other parametric forms that are not parametric F-functions in the design of low-boom targets.

\section{Case Study}

In this section, we demonstrate that Smooth Target 3 in Figure 2, which satisfies the volume requirement for the cockpit, can be matched closely by a configuration. The low-fidelity low-boom design process is essentially the same as the process that is described in reference [12], and the mixed-fidelity process is the same as that described in reference [2]. The key differences are that the geometry format is more flexible for modeling more complicated 
shapes and the logic node in the ModelCenter flow process is used to switch among analyses with varying fidelities. See reference [13] for the updated multifidelity supersonic analysis and design process.

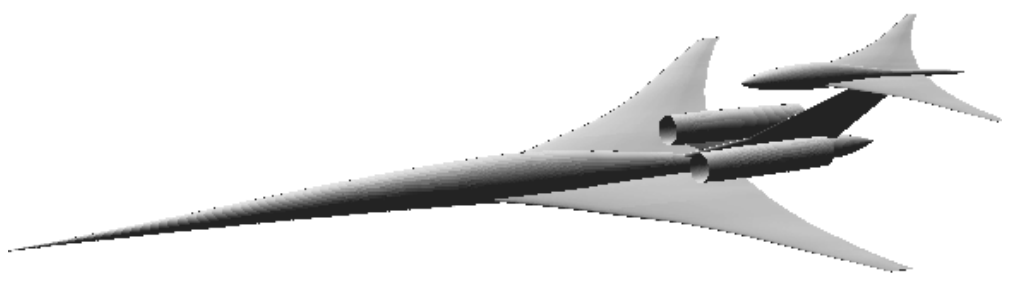

Figure 4. Mixed-fidelity design.

The low-boom configuration is shown in Figure 4. It is designed for a cruise weight of 30,000 lb, a cruise Mach number of 1.6, and a cruise altitude of 45,000 ft. The configuration has the following dimensions: the length is 127 $\mathrm{ft}$, the span is $39 \mathrm{ft}$, and the height is $14 \mathrm{ft}$. The wing reference area is $688 \mathrm{ft}^{2}$, and the cruise lift coefficient is 0.08 at an angle of attack of 0.5 degrees. The equivalent length of the $A_{\mathrm{e}}$ of the configuration is $140 \mathrm{ft}$, and the $A_{\mathrm{e}}$ value at $x$ $=140$ is $33.8 \mathrm{ft}^{2}$. This configuration was inspired by a public configuration of the Gulfstream low-boom demonstrator; however, the nacelle geometry was generated by NPSS [14].

Smooth Target 3 in Figure 2 was chosen as the target for this low-boom configuration because of the cockpit volume constraint. After the initial concept exploration with a low-fidelity analysis, all the components except the fuselage and the aft pod were fixed. Then, BOSS [12] was used to reshape the fuselage and the aft pod to match the low-fidelity $A_{\mathrm{e}}$ of the configuration to the target $A_{\mathrm{e}}$. Figure 5 shows an exceptionally good matching of the target $A_{\mathrm{e}}$ by the low-fidelity low-boom configuration.
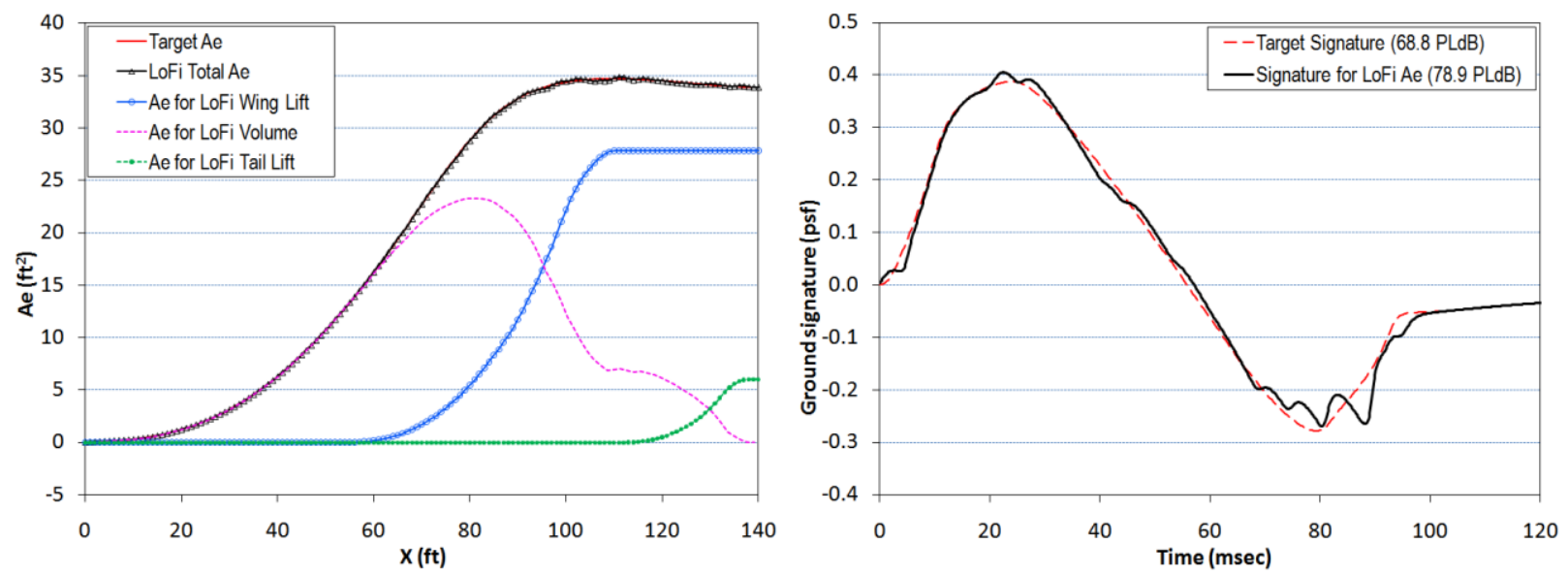

Figure 5. Low-fidelity boom analysis of low-boom baseline.
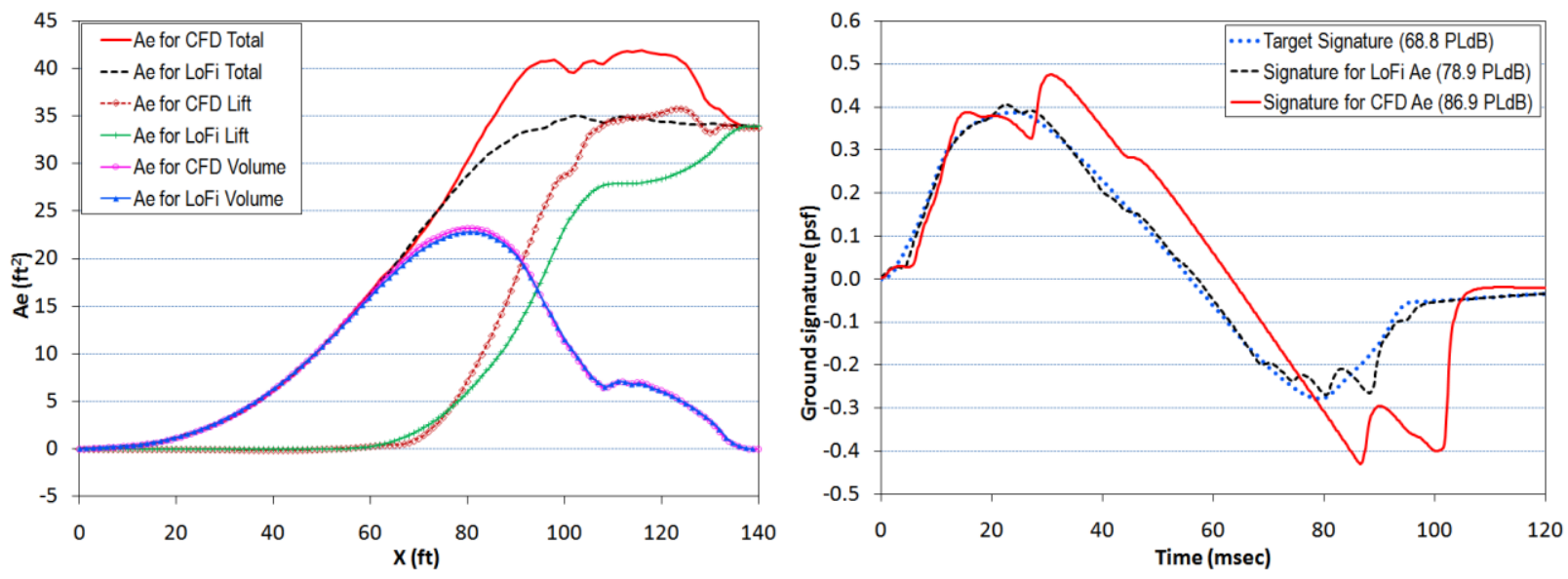

Figure 6. CFD $A_{\mathrm{e}}$ analysis of low-fidelity low-boom baseline. 
Then, CFD $A_{\mathrm{e}}$ analysis of the low-fidelity design was used to reveal a discrepancy between the low- and highfidelity $A_{\mathrm{e}}$ analyses. Because the downwash of the wing on the horizontal tail is not captured in the low-fidelity analysis, the horizontal tail lift from the CFD analysis is almost zero (see the $A_{\mathrm{e}}$ for CFD lift in Figure 6). The tail lift difference results in a significant mismatch between the CFD $A_{\mathrm{e}}$ and the target $A_{\mathrm{e}}$, as well as an increase of 8 $\mathrm{PLdB}$ in the ground signature that is propagated from the CFD $A_{\mathrm{e}}$.
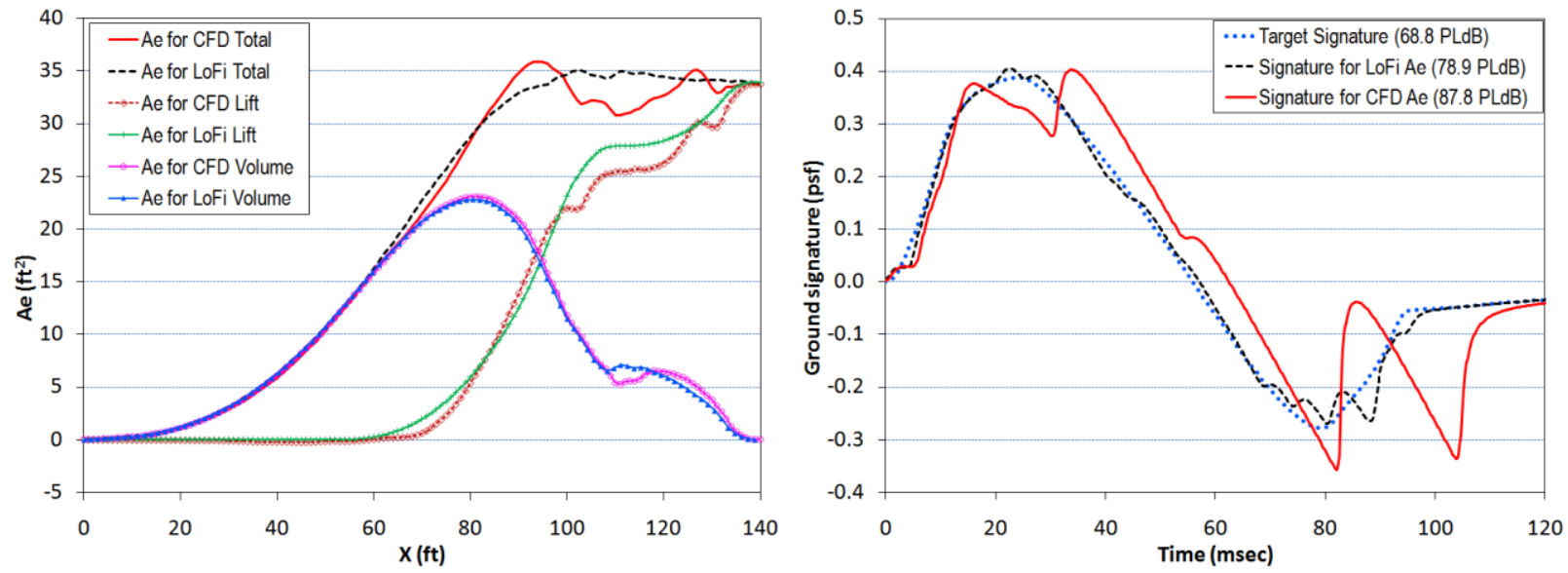

Figure 7. $A_{\mathrm{e}}$ analysis of the modification for CFD tail lift adjustment.

Tail lift was added by adjusting the incidence of the horizontal tail until the resulting CFD $A_{\mathrm{e}}$ distribution for the configuration was close enough to the target $A_{\mathrm{e}}$ for fuselage and pod shaping. Figure 7 shows the low-fidelity analysis results for the low-fidelity low-boom baseline and the CFD analysis results for the configuration with the tail lift adjustment. Note that the low- and high-fidelity volume $A_{\mathrm{e}}$ difference is relatively small, mainly due to the adjustment in the incidence for the horizontal tail, while the $A_{\mathrm{e}}$ for the CFD lift is much closer to the $A_{\mathrm{e}}$ for the lowfidelity lift. After no more than ten iterations of the mixed-fidelity low-boom design process [2], we were able to reduce the discrepancy between the CFD $A_{\mathrm{e}}$ for the configuration and the target $A_{\mathrm{e}}$, as shown in the right plot of Figure 8 .
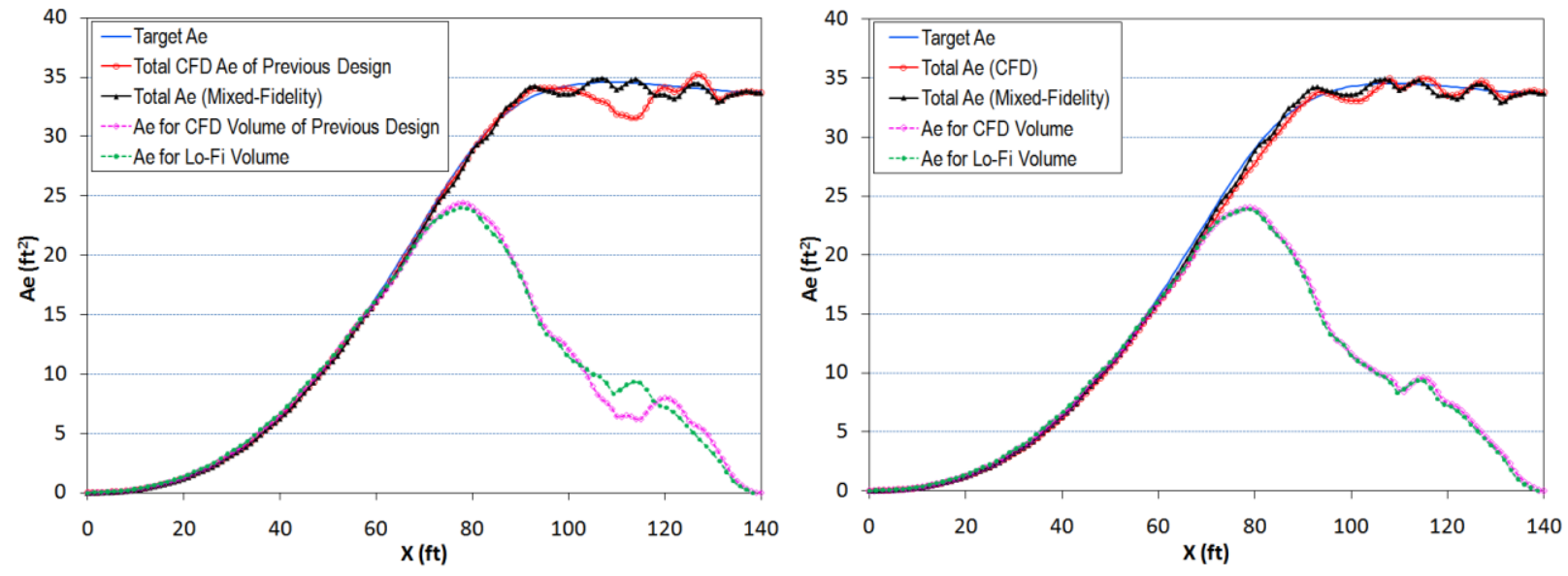

Figure 8. $A_{\mathrm{e}}$ analysis of mixed-fidelity design.

Recall that the mixed-fidelity approach uses the following approximation of the actual CFD $A_{\mathrm{e}}$ of the configuration:

$$
A_{e}^{\text {mixed }}=A_{e, C F D}^{\text {total }}-A_{e, \text { old }}^{\text {volume }}+A_{e, \text { new }}^{\text {volume }}
$$


where the total CFD $A_{\mathrm{e}}$ is for the previous design, the old volume $A_{\mathrm{e}}$ is the low-fidelity volume for the previous design, and the new volume $A_{\mathrm{e}}$ is the low-fidelity volume for the mixed-fidelity design.

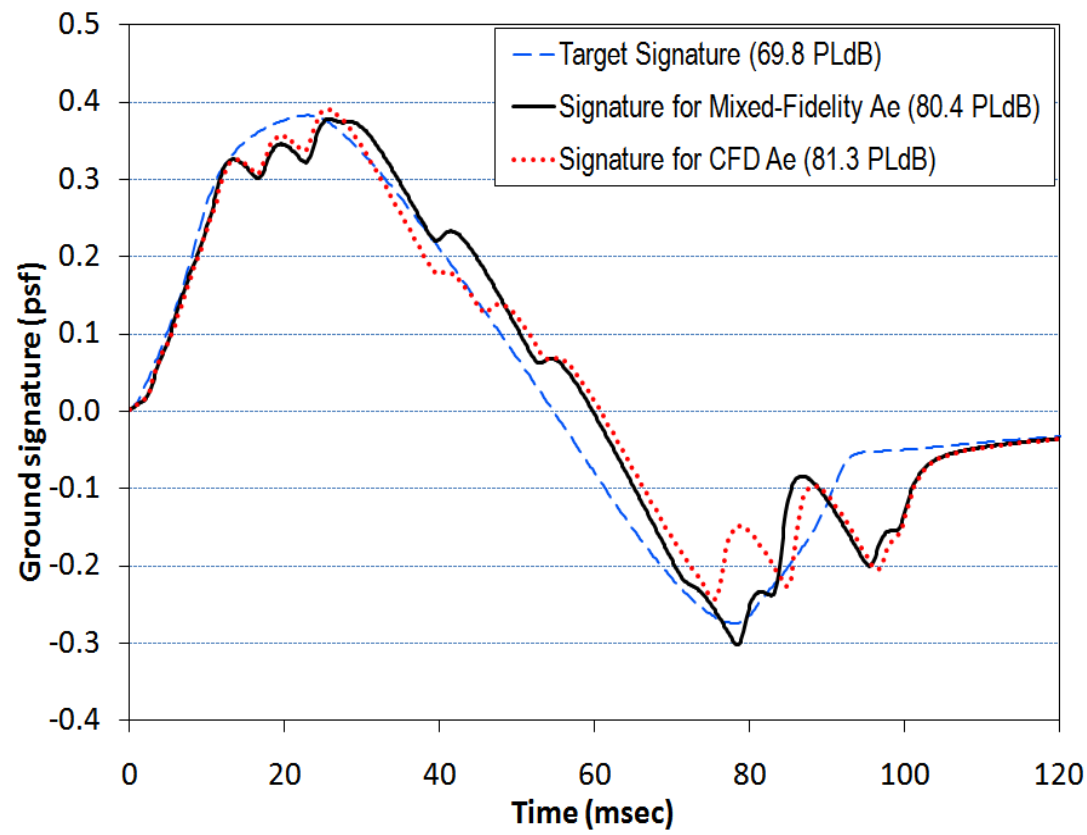

Figure 9. Comparison of signatures for mixed-fidelity design.

The key idea in the mixed-fidelity approach is to use the low-fidelity $A_{\mathrm{e}}$ analysis during the $A_{\mathrm{e}}$ matching to obtain a configuration that has a ground signature with low-boom characteristics (such as a shaped signature and a lower PLdB value). The final iteration of the mixed-fidelity low-boom shaping yields a fairly good match to the target $A_{\mathrm{e}}$ (see the left plot in Figure 8 ). The signature that is propagated from the mixed-fidelity $A_{\mathrm{e}}$ has a ramp-type shape in the front portion and some shaping in the aft portion, with a PLdB value of 80.4 (see Figure 9). The difference of 1 PLdB between the low-fidelity and the CFD target signature is mainly due to the difference of 0.15 between the two target $A_{\mathrm{e}}$ values at $x=140$, which is caused by numerical errors in matching the total cruise lift. The CFD $A_{\mathrm{e}}$ analysis of the mixed-fidelity design shows the accuracy of the mixed-fidelity analysis results (see the right plot in Figure 8, as well as Figure 9). Further mixed-fidelity iterations failed to improve the $A_{\mathrm{e}}$ matching substantially or yield a better ground signature. As the result, the mixed-fidelity process was terminated.

To verify that the mixed-fidelity design has a shaped ground signature, a CFD off-body pressure distribution is generated by using the method that is documented in reference [8]. Figure 10 shows the $d p / p$ at three body lengths below the configuration and the corresponding ground signature.
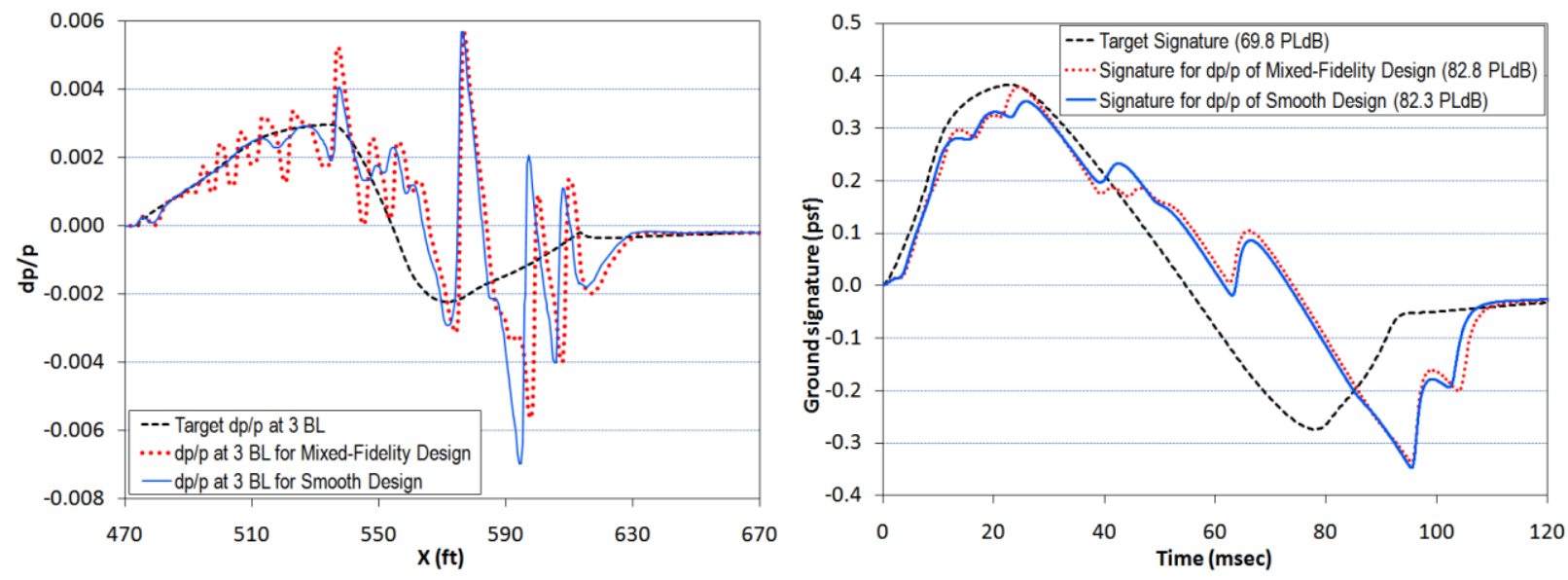

Figure 10. Off-body $d p / p$ and signatures for mixed-fidelity design and smooth design. 

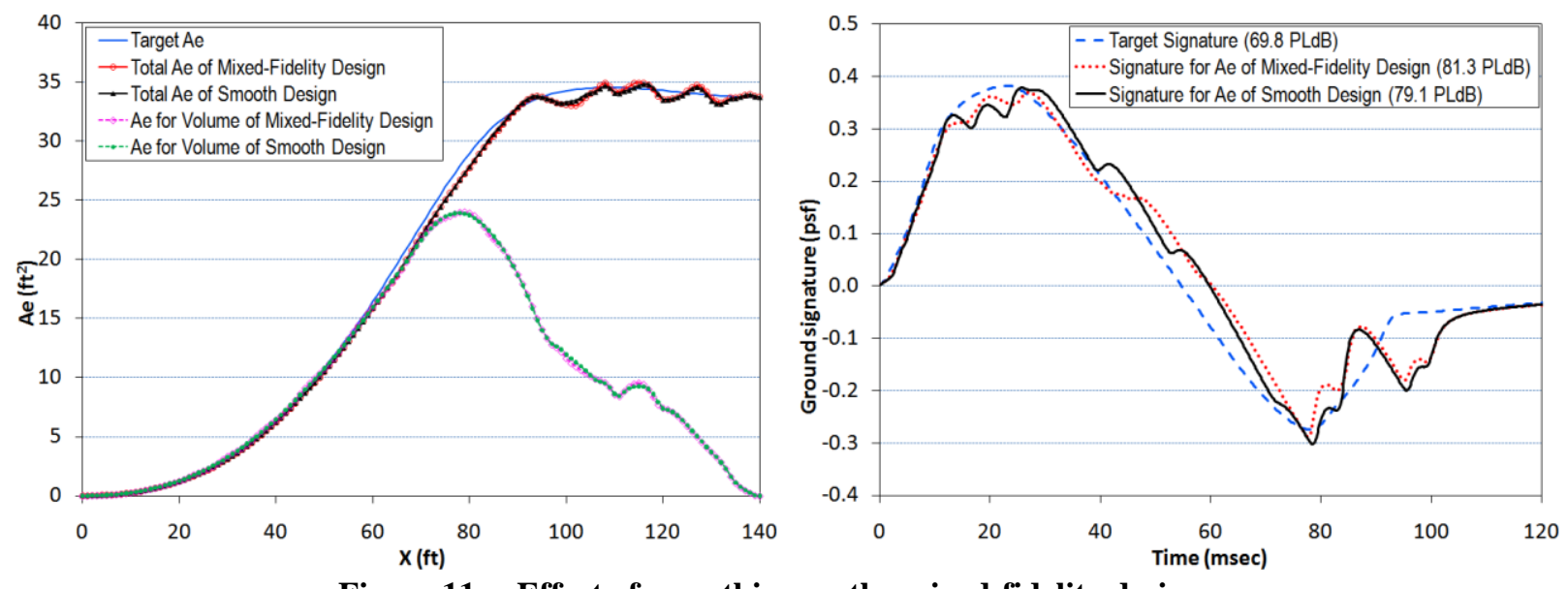

Figure 11. Effect of smoothing on the mixed-fidelity design.

Note that the front portion of the $d p / p$ at three body lengths below the configuration is not as smooth as the target $d p / p$ that is propagated from the target $A_{\mathrm{e}}$. These peaks and valleys are due to oscillations in the derivatives of the camber line and the derivatives of the cross-section area distribution of the fuselage, which are caused by fuselage shaping. Therefore, we smoothed the fuselage and the pod of the mixed-fidelity design. The resulting differences in these two configurations are shown in Figure 11. Some small changes in the CFD $A_{\mathrm{e}}$ lead to a reduction of 2 PLdB in the perceived loudness of the ground signature. The off-body pressure distribution and the corresponding signature of the smooth design are shown in Figure 10. Even though there is no significant difference in the ground signatures of these two designs (see the right plot of Figure 10), the exceptionally good match of the front portion of $d p / p$ for the smooth design and that of the target $d p / p$ makes the smooth design a better candidate for $d p / p$ tailoring to further improve the design.

\section{Concluding Remarks}

A parametric $A_{\mathrm{e}}$ target explorer was developed to generate a low-boom $A_{\mathrm{e}}$ target while satisfying the $A_{\mathrm{e}}$ volume requirements of a configuration. The interactive Excel interface of the $A_{\mathrm{e}}$ target explorer allows users to conduct tradeoff analyses between $A_{\mathrm{e}}$ volume requirements and low-boom characteristics (e.g., the perceived loudness level) of the corresponding ground signature. The $A_{\mathrm{e}}$ target is parameterized by the $x$ and $y$ coordinates of the control points of a B-spline curve. As a result, the $A_{\mathrm{e}}$ volume requirements can easily be satisfied by specifying lower and upper bounds on the $y$ coordinates of the control points. This allows users to generate a feasible $A_{\mathrm{e}}$ target by minimizing the PLdB value of the target signature with only bounds on the control points.

To demonstrate the feasibility of the generated target, a case study was presented to match the $A_{\mathrm{e}}$ target with a configuration that consists of a fuselage, wing, pylon, nacelle, aft pod, vertical tail, and horizontal tail. The case study shows that the generated $A_{\mathrm{e}}$ target can be matched exceptionally well with a configuration by using a lowfidelity $A_{\mathrm{e}}$ analysis. A CFD $A_{\mathrm{e}}$ analysis revealed, however, that low-fidelity lift analysis is not adequate to capture flow interactions between the wing, the horizontal tail, and other components. Adjustment of the incidence of the horizontal tail can be used to make the $A_{\mathrm{e}}$ for the CFD lift similar to the $A_{\mathrm{e}}$ for the low-fidelity lift. A low-boom configuration was obtained by using the mixed-fidelity CFD $A_{\mathrm{e}}$ matching process. This demonstrates that the mixedfidelity design process is an effective approach for low-boom design. The sonic-boom analysis that was conducted using the off-body pressure distribution at three body lengths below the configuration was used to verify the mixedfidelity design. The final design had a shaped front signature with a PLdB value of 82.3. The aft portion of the ground signature was not an $\mathrm{N}$-wave but was not as shaped as the target; thus, further improvement of the aft shape of the ground signature may require a design method that is capable of tailoring off-body $d p / p$ distributions for lowboom design. 


\section{References}

[1] ModelCenter Ver. 6.0, Design Integration Software, Phoenix Integration, Blacksburg, VA, 2004.

[2] Li, W., Shields, E., and Geiselhart, K., "A Mixed-Fidelity Approach for Design of Low-Boom Supersonic Aircraft,” AIAA-2010-0845, January 2010.

[3] Seebass, R., and George, A., "Sonic-Boom Minimization," Journal of the Acoustical Society of America, Vol. 51, No. 2, 1972, pp. 686-694.

[4] Darden, C., "Sonic Boom Minimization with Nose-Bluntness Relaxation,” NASA TP-1348, 1979.

[5] Mack, R., and Haglund, G., "Practical Low-Boom Overpressure Signature Based on Minimum Sonic Boom Theory," High-Speed Research: Sonic Boom: Vol. 2, edited by C. Darden, NASA-CP-3173, Feb. 1992, pp. 1530.

[6] Plotkin, K., Rallabhandi, S., and Li, W., "Generalized Formulation and Extension of Sonic Boom Minimization Theory for Front and Aft Shaping." AIAA-2009-1052, January 2009.

[7] Seebass, R., and Argrow, B., "Sonic Boom Minimization Revisited," AIAA-1998-2956, June 1998.

[8] Ordaz, I., and Li, W., "Integration of Off-Track Sonic Boom Analysis in Conceptual Design of Supersonic Aircraft," AIAA paper no. pending, January 2011.

[9] Plotkin, K., "Review of Sonic Boom Theory," AIAA-1989-1105, April 1989.

[10] Rallabhandi, S., "Advanced Sonic Boom Prediction Using Augmented Burger's Equation," AIAA paper no. pending, January 2011.

[11] Howe, D., "Improved Sonic Boom Minimization with Extended Nose Spike," AIAA-2005-1014, January 2005.

[12] Li, W., Shields, E., and Le, D., "Interactive Inverse Design Optimization of Fuselage Shape for Low-Boom Supersonic Concepts," Journal of Aircraft, Vol. 45, No. 4, 2008, pp. 1381-1398.

[13] Geiselhart, K., Ozoroski, L., Fenbert, J., Shields, E., and Li, W., "Integration of Multifidelity Multidisciplinary Computer Codes for Design and Analysis of Supersonic Aircraft," AIAA paper no. pending, January 2011.

[14] Lytle, J., Follen, G., Naiman, C., Evans, A., Veres, J., Owen, K., and Lopez, I., "Numerical Propulsion System Simulation (NPSS) 1999 Industry Review," NASA-TM-209795, August 2000. 